\title{
estualio de la influencia que los distintos tipos
de refrentado ejereen en el ensayo a compresión
de probetas de hormigón
}

A. DELIBES LINIERS, Ingeniero de Caminos * G. GONZALEZ ISABEL, Ingeniero Técnico C. C. *

\begin{abstract}
sinopsis
Se exponen los resultados del estudio experimental de distintas técnicas de refrentado de probetas de hormigón, todas ellas admitidas generalmente como correctas. Se observan diferencias notables de unos sistemas a otros, recomendándose finalmente el empleo de azufre fundido para hormigones hasta $500 \mathrm{kp} / \mathrm{cm}^{2}$ de resistencia. Se establecen también algunas normas de ejecución que no aparecen en las distintas especificaciones vigentes.
\end{abstract}

\section{INTRODUCCION}

El objetivo que se pretende con el presente plan de ensayos es estudiar la influencia que los distintos tipos de acabado o preparación de la cara superior de las probetas moldeadas de hormigón ejercen en los resultados del ensayo a compresión de probetas cilíndricas de hormigón. Como es sabido, la correcta ejecución de esta operación, denominada refrentado, tiene una influencia decisiva en dichos resultados. Un refrentado defectuoso conducirá, en muchos casos, a calificar el hormigón de forma muy desfavorable. Así, un error inapreciable $(0,25 \mathrm{~mm})$ en la cara de acabado puede reducir la resistencia de la probeta en más de un $30 \%$.

Los tipos de refrentado que recogen los ensayos son los que emplean polvo de cemento, pasta pura de cemento, mortero de azufre y azufre puro. Unicamente el primero de ellos se realiza sobre el hormigón de la probeta aún fresco. En todos los casos se supone que se trata de operaciones correctamente ejecutadas de acuerdo con la normativa actual.

Se han descartado otros sistemas, como pueden ser la pasta de yeso o el mortero del mismo material, el pulido con muela abrasiva
- la interposición de tapas de cartón o material similar, entre la cara de acabado de las probetas y el plato de la prensa.

\section{COMENTARIOS A LOS DISTINTOS SISTEMAS DE REFRENTADO}

Los factores comunes en que deben coincidir todos los materiales o sistemas de refrentado de probetas de hormigón, son de conocimiento casi general y podemos resumirlos en los puntos siguientes:

- Existen unas tolerancias relativas a la planeidad y perpendicularidad al eje de las probetas de hormigón, que para su cumplimiento hacen necesario el refrentado de la cara de acabado.

- El espesor del refrentado debe ser mínimo, estando acotado superiormente por las normas en vigor.

- La resistencia del material de refrentado debe ser superior a la del hormigón de la probeta.

- La influencia del refrentado es mayor en hormigones de alta resistencia.

* Del Instituto Técnico de Materiales y Construcciones (INTEMAC). 
- Para la dosificación de materiales de refrentado y su ejecución se siguen en líneas generales las especificaciones UNE $7242 y$, en su defecto, las A.F.N.O.R o A.S.T.M. correspondientes.

\section{PLAN DE ENSAYOS}

\section{Variables en estudio}

\section{Resistencias del hormigón}

El estudio se ha realizado sobre los cuatro hormigones-tipo siguientes:

$$
\begin{aligned}
& \text { H-125 } \\
& \text { H-250 } \\
& \text { H-350 } \\
& \text { H-450 }
\end{aligned}
$$

\section{Tipos de refrentado}

Se han empleado cuatro tipos distintos en cuanto a su composición:
a) Polvo de cemento
b) Azufre puro
c) Mortero de azufre
d) Pasta pura

\section{Fabricación del hormigón}

El hormigón ha sido fabricado en el Laboratorio Central de INTEMAC en una amasadora centrífuga planetaria TEKA, con arreglo a las siguientes dosificaciones expresadas en peso de los componentes y referidas a $1 \mathrm{~m}^{3}$ de hormigón:

\section{$\mathrm{H}-125$}

$\begin{array}{lllllrl}\text { Grava } 5 / 18 \mathrm{~mm} & \ldots & \ldots & \ldots & 1.300 \mathrm{~kg} \\ \text { Arena } 0 / 5 \mathrm{~mm} & \ldots & \ldots & \ldots & \ldots & 700 \mathrm{~kg} \\ \text { Cemento P-350 } & \ldots & \ldots & \ldots & \ldots & 250 \mathrm{~kg} \\ \text { Agua añadida } & \ldots & \ldots & \ldots & \ldots & 200 \mathrm{l} \\ \text { (Relación a/c } & \ldots & \ldots & \ldots & \ldots & 0,800 \text { ) } \\ \text { (Asiento de cono } & \ldots & \ldots & \ldots & 10 \mathrm{~cm} \text { ) }\end{array}$

\begin{tabular}{|c|c|}
\hline ava $5 / 18$ & 200 \\
\hline a $0 / 5 \mathrm{~mm} \ldots$ & 650 \\
\hline ento P-350 .. & 425 \\
\hline$\ldots \ldots$ & 210 \\
\hline & \\
\hline 10 & \\
\hline
\end{tabular}

\section{H-250}

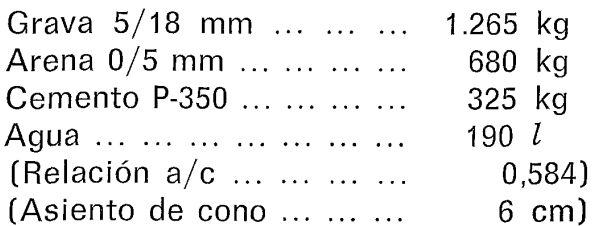

\section{$H-350$}

\section{H-450}

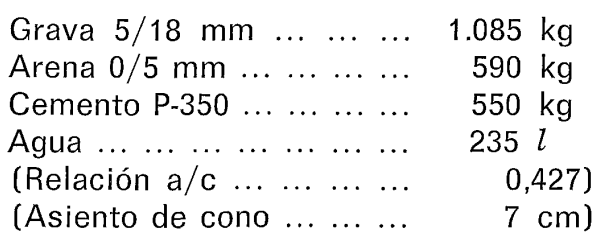

\section{Fabricación de las probetas}

La fabricación de las probetas ha sido reali. zada en el interior de una cámara con $65 \%$ de H.R. y $20^{\circ} \mathrm{C}$ de temperatura.

A partir de una amasada de $350 l$ se fabricaron para cada resistencia de hormigón un total de 16 probetas cilíndricas $15 \times 30 \mathrm{~cm}$, distribuyéndolas en grupos de 4 para cada uno de los tipos de refrentado en estudio.

El Ilenado de los moldes se ha hecho en dos tongadas de $15 \mathrm{~cm}$ cada una. Se han compactado empleando un vibrador con diámetro de aguja de $20 \mathrm{~mm}$ y frecuencia de 9.000 r.p.m.

\section{Curado y conservación de las probetas}

Una vez fabricadas las probetas, sus superficies libres fueron enrasadas con un fratás de madera, de forma que no quedasen desigualdades superiores a $1 \mathrm{~mm}$. A continuación se cubrieron las superficies libres con arpillera empapada en agua y sujetas con una goma a la cabeza del molde, envolviendo la probeta en una bolsa de plástico, la cual se cerró herméticamente.

A partir de este momento, y durante las siguientes 24 horas, los moldes no sufrieron ningún tipo de manipulación. Las probetas destinadas a refrentado en fresco con polvo de cemento, reciben un tratamiento especial como se indica más adelante.

Pasadas 24 horas las probetas fueron desmoldadas y trasladadas a la cámara de curado (H.R. $\left.=100 \% ; \mathrm{T}=21^{\circ} \mathrm{C}\right)$, donde permanecieron hasta 3 horas antes del momento del ensayo. 


\section{Refrentado de las probetas}

Se indican a continuación los distintos sistemas seguidos para el refrentado, que, en general, siguen las especificaciones de las Normas A.S.T.M. y A.F.N.O.R. correspondientes.

\section{Refrentado con polvo de cemento}

Este sistema se aplicó a probetas recién fabricadas, por lo que se realizó en el mismo lugar donde se confeccionaron.

Una vez compactada la cara superior se esperó a que comenzase el fraguado (suele apreciarse por la pérdida de brillo del hormigón en su superficie). A continuación se enrasó la cara libre con una regla metálica ancha y rígida, espolvoreando sobre la superficie del hormigón cementos del mismo tipo y categoría que el empleado en la fabricación del hormigón, a razón de $1,7 \mathrm{~g} / \mathrm{cm}^{2}$ (300 g por probeta), pasando la regla apoyada en la boca del molde repetidas veces en todas las direcciones, hasta conseguir una cara perfectamente lisa y plana. Una vez realizado el refrentado se colocó el molde en bolsa de plástico cerrada y se conservó en estas con diciones hasta el momento del desmoldeo y la introducción en la cámara de curado.

Hay que hacer notar que según las Normas A.S.T.M. C-31 y C-192, el acabado debe hacerse con un plato de vidrio o acero pulido; sin embargo, este sistema puede acarrear dificultades de ejecución al adherirse la pasta al plato, según nuestra opinión y experiencia previa.

\section{Refrentado con pasta pura}

La pasta pura se ha fabricado con cemento P-350 y una relación agua/cemento de 0,37. Antes de utilizar la pasta se esperaron 4 horas para que al emplearla hubiese sufrido su retracción inicial. A continuación se extendió la pasta con la regla, pasándola en todas las direcciones, hasta obtener una cara perfectamente plana y lisa.

Nuestra experiencia es que el refrentado con pasta pura no debe hacerse sobre probetas secas, ya que, entonces, absorberán agua de la pasta pura, agrietándose la capa de refrentado. Por este motivo, el refrentado se hizo inmediatamente después de extraerlas de la cámara, siendo introducidas de nuevo en dicha cámara una vez efectuado. Este tipo de refrentado se llevó a cabo 4 días antes de la fecha de ensayo. Se empleó un cemento P-350 en lugar de otro de más rápido fraguado y endurecimiento, pues teníamos antecedentes de importantes agrietamientos de la pasta, muy dífíciles de evitar empleando cemento de alta resistencia inicial. Por lo demás, no se presentan problemas de baja resistencia, al menos en términos relativos. (Véanse los resultados de ensayo de materiales de refrentado.)

\section{Refrentado con mortero de azufre}

El mortero de azufre empleado tenía la siguiente composición, en peso:

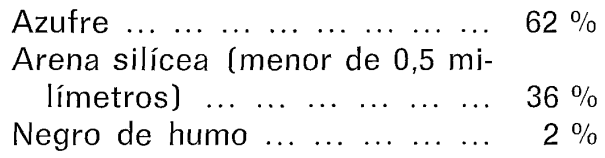

Se utilizó un recipiente para fundir el azufre equipado con control automático de temperatura para mantener ésta en $130^{\circ} \mathrm{C}$.

Desde que se sacaron las probetas de la cámara se esperó a que la superficie de las probetas no estuviese excesivamente húmeda, con el fin de que el refrentado adheriese bien y no se formasen burbujas de vapor (aproximadamente media hora en ambiente con humedad normal).

\section{Refrentado con azufre}

Se realizó con azufre puro fundido a $130^{\circ} \mathrm{C}$. Por lo demás, son válidas las consideraciones del apartado anterior.

\section{ENSAYOS DEL MATERIAL DE REFRENTADO}

De los materiales empleados para el refrentado de las probetas de hormigón se efectuaron los ensayos especificados a continuación.

\section{Ensayos de azufre}

Se ensayaron a compresión 6 probetas de azufre de $4 \times 4 \times 4 \mathrm{~cm}$, fabricadas con el material puro y con el mortero de refrentado. La operación de llenado de los moldes es delicada, debiendo extremarse las precauciones para evitar que quede aire ocluido o se produzcan grietas. Las medidas adoptadas entre otras fueron: 
1) Calentar el molde a $60^{\circ} \mathrm{C}$, manteniéndolo a esta temperatura.

2) Pintar con aceite todas las caras.

3) Rellenar por capas de $3 \mathrm{~mm}$ de espesor máximo, permitiendo el enfriamiento y consiguiente solidificación de la capa anterior antes de proceder al vertido de la siguiente.

La edad de ensayo de estas probetas fue idéntica a la del material de refrentado de las cilíndricas (2 horas).

\section{Pasta pura}

Se ensayaron igualmente 6 probetas de $4 \times 4 \times 4 \mathrm{~cm}$, fabricadas con el material em pleado para el refrentado. La edad en el momento del ensayo era de 4 días.

\section{Resultados}

Los resultados obtenidos figuran en el cuadro siguiente:

\section{RESISTENCIA A COMPRESION DE LOS MATERIALES DE REFRENTADO MEDIDA EN PROBETAS DE $4 \times 4 \times 4 \mathrm{~cm}$}

\begin{tabular}{|c|c|c|c|c|}
\hline $\begin{array}{l}\text { PRODUCTO } \\
\text { EMPLEADO }\end{array}$ & $\begin{array}{c}\text { EDAD } \\
\text { AL } \\
\text { ENSAYO }\end{array}$ & $\begin{array}{c}\text { DESIGNACION } \\
\text { OE LA } \\
\text { PROBETA }\end{array}$ & $\stackrel{f}{f}$ & $\begin{array}{c}\mathbf{f}_{m} \\
{\left[\mathrm{kp}_{\mathrm{m}} / \mathrm{cm}^{2}\right]}\end{array}$ \\
\hline $\begin{array}{c}\text { AZUFRE } \\
\text { PURO }\end{array}$ & 2 horas & $\begin{array}{l}1 \mathrm{~A} \\
2 \mathrm{~A} \\
3 \mathrm{~A} \\
4 \mathrm{~A} \\
5 \mathrm{~A} \\
6 \mathrm{~A}\end{array}$ & $\begin{array}{l}524 \\
525 \\
485 \\
528 \\
495 \\
501\end{array}$ & 510 \\
\hline $\begin{array}{c}\text { MORTERO } \\
\text { DE } \\
\text { AZUFRE }\end{array}$ & 2 horas & $\begin{array}{l}1 M \\
2 M \\
3 M \\
4 M \\
5 M \\
6 M\end{array}$ & $\begin{array}{l}558 \\
531 \\
491 \\
475 \\
549 \\
532\end{array}$ & 523 \\
\hline $\begin{array}{l}\text { PASTA } \\
\text { PURA }\end{array}$ & 4 dias & $\begin{array}{ll}1 & p \\
2 & p \\
3 & p \\
4 & p \\
5 & p \\
6 & p\end{array}$ & $\begin{array}{l}546 \\
574 \\
528 \\
485 \\
490 \\
527\end{array}$ & 525 \\
\hline
\end{tabular}

\section{METODO DE ENSAYO}

Las probetas fueron ensayadas en PRENSA AMSLER de compresión, con capacidad de 100 t, provista de un cadenciómetro incorporado para regular la velocidad de aplicación de la carga. Esta se mantuvo constante hasta rotura con un incremento medio de $6 \mathrm{kp} / \mathrm{cm}^{2} \times \mathrm{s}$

\section{RESULTADOS DE LOS ENSAYOS}

Se indican en las tablas 1 a 4 los resultados de ensayo de las distintas probetas $\left(\boldsymbol{f}_{c}\right)$, los valores medios $\left(\boldsymbol{f}_{\mathrm{cm}}\right)$ y los valores de la desviación cuadrática media relativa (V) obtenida en cada caso. En las figuras 1 a 4 se expresan estos resultados medios en porcentaje del más elevado de ellos, para cada uno de los hormigones-tipo. Finalmente, en la figura 5 , se expresan en conjunto estos resultados, en función del valor de la resistencia del hormigón $\left(\mathbf{f}_{\mathrm{cm}}\right)$ obtenido en cada caso.

\section{DISCUSION DE LOS RESULTADOS DE ENSAYO}

Todos los materiales empleados en el refrentado sobre probeta endurecida tienen resistencias muy similares para las edades en que se utilizan. Estas son del orden de los $500 \mathrm{kp} / \mathrm{cm}^{2}$.

La ejecución del refrentado con polvo de cemento requiere la utilización de la boca del molde como apoyo de referencia para el útil empleado en la terminación. Esto obligará a llevar un control muy riguroso del estado de los moldes, lo cual puede resultar laborioso. En esta investigación, mediante el empleo de moldes totalmente nuevos, se ha conseguido una uniformidad de resultados muy satisfactoria.

En la figura 5 puede observarse lo siguiente:

a) El refrentado con azufre puro o mortero de azufre conduce a resultados prácticamente idénticos, siendo los más favorables en todos los casos.

b) El refrentado con pasta pura de cemento P-350 da lugar a análogos descensos de resistencia (del orden del $10 \%$ ) en todos los casos. 
TABLA 1 HORMIGON TIPO $\mathrm{H}-125$

\begin{tabular}{|c|c|c|c|c|c|}
\hline $\begin{array}{c}\text { PRODUCTO } \\
\text { DE } \\
\text { REFRENTADO }\end{array}$ & $\begin{array}{c}\text { DESIGNACION } \\
\text { DE LAS } \\
\text { PROBETAS }\end{array}$ & $\begin{array}{c}\text { EDAD } \\
\text { AL } \\
\text { ENSAYO }\end{array}$ & $\begin{array}{c}f_{c} \\
\left(k_{p} / \mathrm{cm}^{2}\right)\end{array}$ & $\underset{\left.\mathrm{ckp} / \mathrm{cm}^{2}\right]}{f_{c m}}$ & $\begin{array}{l}v \\
\%\end{array}$ \\
\hline $\begin{array}{c}\text { POLVO } \\
\text { DE } \\
\text { CEMENTO }\end{array}$ & $\begin{array}{l}1 C \mathrm{~A} \\
1 \mathrm{C} \mathrm{B} \\
1 \mathrm{CC} \\
1 \mathrm{C} D\end{array}$ & 28 días & $\begin{array}{l}128 \\
132 \\
129 \\
131\end{array}$ & 130 & 1,2 \\
\hline $\begin{array}{l}\text { PASTA } \\
\text { PURA }\end{array}$ & $\begin{array}{l}1 P A \\
1 P B \\
1 P C \\
1 P D\end{array}$ & 28 días & $\begin{array}{l}121 \\
124 \\
125 \\
123\end{array}$ & 123 & 1,2 \\
\hline AZUFRE & $\begin{array}{l}1 \mathrm{AA} \\
1 \mathrm{AB} \\
1 \mathrm{AC} \\
1 \mathrm{AD}\end{array}$ & 28 días & $\begin{array}{l}128 \\
131 \\
132 \\
134\end{array}$ & 131 & 1,7 \\
\hline $\begin{array}{l}\text { MORTERO } \\
\text { DE } \\
\text { AZUFRE }\end{array}$ & $\begin{array}{l}1 M A \\
1 M B \\
1 M C \\
1 M D\end{array}$ & 28 dias & $\begin{array}{l}132 \\
135 \\
131 \\
140\end{array}$ & 135 & 2,6 \\
\hline
\end{tabular}

TABLA 2 HORMIGON TIPO $\quad \mathrm{H}-250$

\begin{tabular}{|c|c|c|c|c|c|}
\hline $\begin{array}{c}\text { PRODUCTO } \\
\text { DE } \\
\text { REFRENTADO }\end{array}$ & $\begin{array}{c}\text { DESIGNACION } \\
\text { DE LAS } \\
\text { PROBETAS }\end{array}$ & $\begin{array}{c}\text { EDAD } \\
\text { AL } \\
\text { ENSAYO }\end{array}$ & $\begin{array}{c}f_{c} \\
{\left[\mathrm{kp}_{\mathrm{p}} / \mathrm{cm}^{2}\right]}\end{array}$ & $\begin{array}{c}f_{\mathrm{cm}} \\
\left(\mathrm{kp}_{\mathrm{p}} / \mathrm{cm}^{2}\right)\end{array}$ & $\begin{array}{l}\mathbf{v} \\
\%\end{array}$ \\
\hline $\begin{array}{l}\text { POLVO } \\
\text { DE } \\
\text { CEMENTO }\end{array}$ & $\begin{array}{l}2 \text { C A } \\
2 \text { C B } \\
2 \text { C C } \\
2 \text { C D }\end{array}$ & 28 días & $\begin{array}{l}259 \\
257 \\
268 \\
258\end{array}$ & 261 & 1,7 \\
\hline $\begin{array}{l}\text { PASTA } \\
\text { PURA }\end{array}$ & $\begin{array}{l}2 P A \\
2 P B \\
2 P C \\
2 P D\end{array}$ & 28 días & $\begin{array}{l}246 \\
263 \\
256 \\
258\end{array}$ & 256 & 2,4 \\
\hline AZUFRE & $\begin{array}{l}2 A A \\
2 A B \\
2 A C \\
2 A D\end{array}$ & 28 dias & $\begin{array}{l}299 \\
293 \\
285 \\
290\end{array}$ & 292 & 1,7 \\
\hline $\begin{array}{l}\text { MORIERO } \\
\text { DE } \\
\text { AZUFRE }\end{array}$ & $\begin{array}{l}2 M A \\
2 M B \\
2 M C \\
2 M D\end{array}$ & 28 dias & $\begin{array}{l}291 \\
278 \\
297 \\
291\end{array}$ & 289 & 2,4 \\
\hline
\end{tabular}

c) El refrentado con polvo de cemento da lugar a descensos de resistencia, respecto al azufre fundido, crecientes con la
TABLA 3 HORMIGON TIPO $\mathrm{H}-350$

\begin{tabular}{|c|c|c|c|c|c|}
\hline $\begin{array}{c}\text { PROOUCTO } \\
\text { DE } \\
\text { REFRENTACO }\end{array}$ & $\begin{array}{c}\text { DESIGNACION } \\
\text { DE LAS } \\
\text { PROBETAS }\end{array}$ & $\begin{array}{c}\text { EOAO } \\
\text { AL } \\
\text { ENSAYO }\end{array}$ & $\begin{array}{c}f_{c} \\
\left(k p / \mathrm{cm}^{2}\right)\end{array}$ & $\begin{array}{c}f_{\mathrm{cm}} \\
{\left[\mathrm{kp}_{\mathrm{p}} / \mathrm{cm}^{2}\right)}\end{array}$ & $\begin{array}{l}y \\
\%\end{array}$ \\
\hline \multirow{4}{*}{$\begin{array}{c}\text { POLVO } \\
\text { DE } \\
\text { CEMENTO }\end{array}$} & 3С A & \multirow{4}{*}{28 dias } & 357 & \multirow{4}{*}{357} & \multirow{4}{*}{1,2} \\
\hline & 3 С B & & 363 & & \\
\hline & $3 \subset c$ & & 351 & & \\
\hline & $3 C D$ & & 356 & & \\
\hline \multirow{4}{*}{$\begin{array}{l}\text { PASTA } \\
\text { PURA }\end{array}$} & $3 P A$ & \multirow{4}{*}{28 dias } & 345 & \multirow{4}{*}{337} & \multirow{4}{*}{2,1} \\
\hline & $3 P B$ & & 343 & & \\
\hline & $3 P C$ & & 330 & & \\
\hline & $3 P D$ & & 330 & & \\
\hline \multirow{4}{*}{ AZUFRE } & 3AA & \multirow{4}{*}{28 días } & 397 & \multirow{4}{*}{384} & \multirow{4}{*}{2,1} \\
\hline & $3 \mathrm{AB}$ & & 380 & & \\
\hline & $3 A C$ & & 375 & & \\
\hline & $3 A D$ & & 382 & & \\
\hline \multirow{4}{*}{$\begin{array}{l}\text { MORIERO } \\
\text { DE } \\
\text { AZUFRE }\end{array}$} & ЗMA & \multirow{4}{*}{28 dias } & 358 & \multirow{4}{*}{372} & \multirow{4}{*}{2,6} \\
\hline & ЗM B & & 384 & & \\
\hline & $3 M C$ & & 376 & & \\
\hline & $3 \mathrm{MD}$ & & 370 & & \\
\hline
\end{tabular}

TABLA 4 HORMIGON TIPO $\mathrm{H}-450$

\begin{tabular}{|c|c|c|c|c|c|}
\hline $\begin{array}{c}\text { PROOUCTO } \\
\text { OE } \\
\text { REFRENTADO }\end{array}$ & $\begin{array}{l}\text { DESIGNACION } \\
\text { DE LAS } \\
\text { PROBETAS }\end{array}$ & $\begin{array}{c}\text { EDAD } \\
\text { AL } \\
\text { ENSAYO }\end{array}$ & $\begin{array}{c}f_{c} \\
\left(k p / c m^{2}\right)\end{array}$ & $\begin{array}{c}f_{\mathrm{cm}} \\
\left(k_{\mathrm{p}} / \mathrm{cm}^{2}\right)\end{array}$ & $\begin{array}{l}v \\
\%\end{array}$ \\
\hline $\begin{array}{c}\text { POLVO } \\
\text { OE } \\
\text { CEMENTO }\end{array}$ & $\begin{array}{l}4 C A \\
4 C B \\
4 C C \\
4 C D\end{array}$ & 28 días & $\begin{array}{l}430 \\
422 \\
424 \\
445\end{array}$ & 430 & 2,1 \\
\hline $\begin{array}{l}\text { PASTA } \\
\text { PURA }\end{array}$ & $\begin{array}{l}4 \mathrm{PA} \\
4 \mathrm{~PB} \\
4 \mathrm{PC} \\
4 \mathrm{PD}\end{array}$ & 28 dias & $\begin{array}{l}461 \\
496 \\
479 \\
484\end{array}$ & 480 & 2,6 \\
\hline AZUFRE & $\begin{array}{l}4 \mathrm{AA} \\
4 \mathrm{AB} \\
4 \mathrm{AC} \\
4 \mathrm{AD}\end{array}$ & 28 dias & $\begin{array}{l}521 \\
522 \\
509 \\
544\end{array}$ & 524 & 2,4 \\
\hline $\begin{array}{c}\text { MORTERO } \\
\text { DE } \\
\text { AZUFRE }\end{array}$ & $\begin{array}{l}4 \mathrm{MA} \\
4 \mathrm{MB} \\
4 \mathrm{MC} \\
4 \mathrm{MD}\end{array}$ & 28 dias & $\begin{array}{l}525 \\
516 \\
531 \\
527\end{array}$ & 525 & 1.0 \\
\hline
\end{tabular}

del hormigón. Estos descensos pueden ser importantes (casi del $20 \%$ ) con probetas de elevada resistencia. 

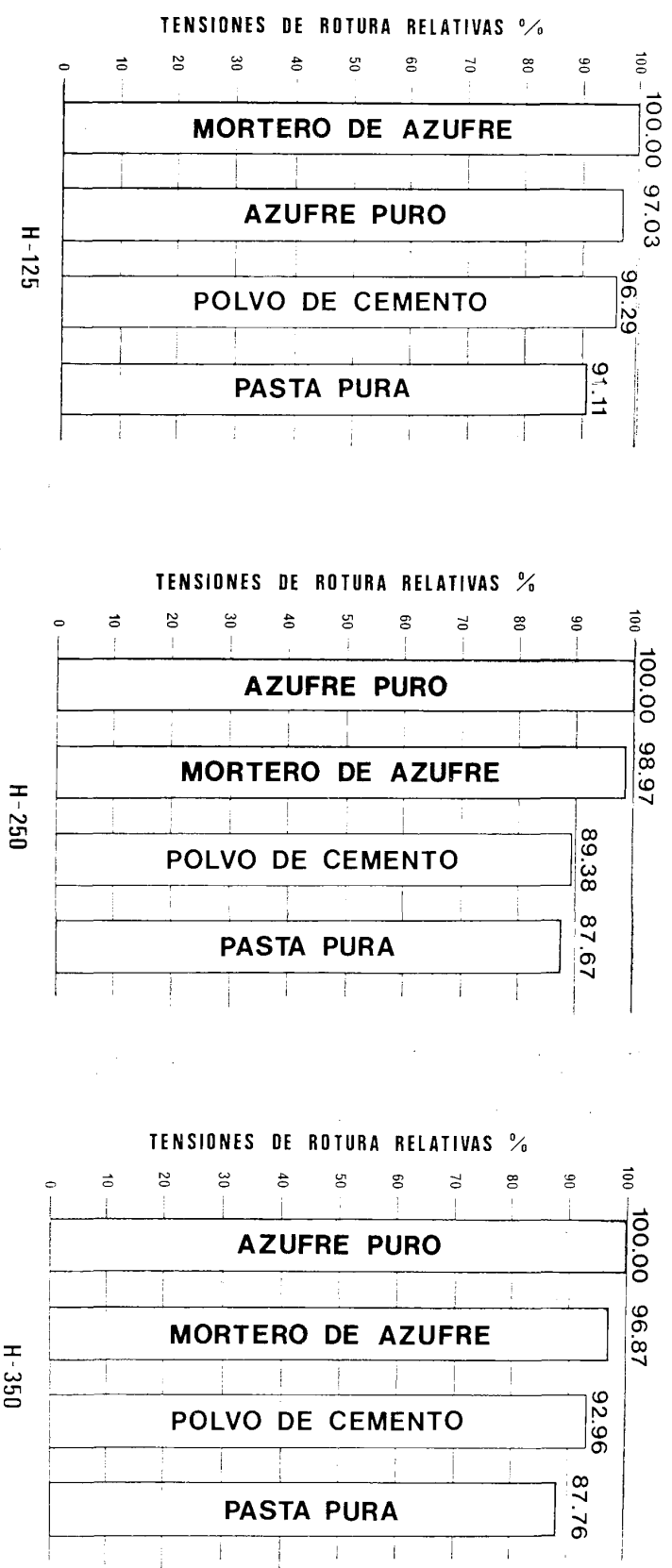

TENSIONES DE ROTURA RELATIVAS \%

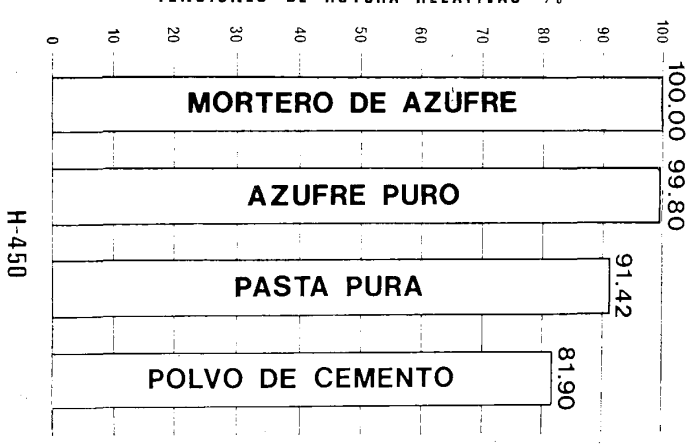

El empleo de pasta pura elaborada con cementos de endurecimiento rápido podría dar lugar a importantes agrietamientos, por lo que, como se ha indicado, fue adoptada una pasta a base de cemento P-350, que a los 4 días alcanzaba una resistencia muy similar a las del azufre fundido. Dicha resistencia ha sido prácticamente igual a la del hormigón de la máxima calidad ensayada. Cabe pensar, por tanto, en la posibilidad de que otras formas de acabado de la superficie de las probetas deben emplearse cuando se ensayan hormigones de resistencia superior a $500 \mathrm{kp} / \mathrm{cm}^{2}$.

Los resultados anteriores coinciden con los obtenidos por otras investigaciones en lo que se refiere a los hormigones de resistencias bajas y medias. No ocurre siempre lo mismo con los de resistencia elevada.

\section{CONCLUSIONES}

Partiendo de la base de que, en las operaciones de acabado de la cara superior de las probetas moldeadas, deben extremarse las precauciones para obtener resultados aceptables, y de acuerdo con lo expuesto, resumiremos brevemente las conclusiones alcanzadas en el estudio de las cuatro técnicas de refrentado más empleadas, todas ellas generalmente admitidas como correctas:

1. El empleo de azufre o mortero de azufre fundido conduce a los resultados más favorables.

2. El empleo de pasta pura de cemento reduce ligeramente las resistencias respecto a las obtenidas con la técnica anterior.

3. El empleo de polvo de cemento sobre el hormigón fresco es delicado y puede dar lugar a bajas de resistencia apreciables $(20 \%)$ cuando es alta la de la probeta.

4. Aunque no se ha experimentado en este estudio, pensamos en la conveniencia de empleo de técnicas más refinadas y costosas cuando hayan de ensayarse probetas de hormigón de resistencia superior a $500 \mathrm{kp} / \mathrm{cm}^{2}$.

5. En todos los casos, es decisiva la cuidadosa ejecución de los trabajos, de acuer. do con lo señalado en el apartado "Refrentado de las probetas". 


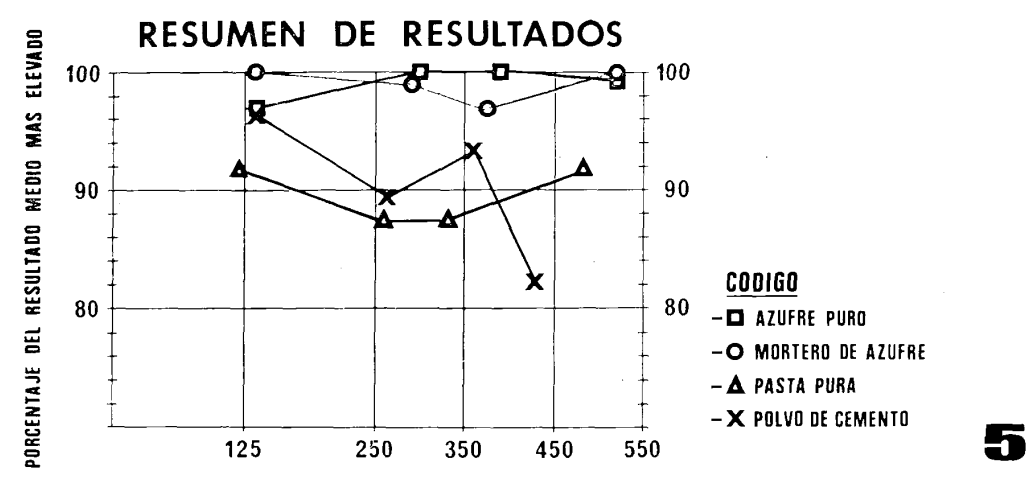

Los ensayos analizados se han llevado a cabo en el Laboratorio Central de INTEMAC durante el año 1975, tras haber experimentado las distintas técnicas descritas.

\section{BIBLIOGRAFIA}

1. U. S. BUREAU OF RECLAMATION: "Concrete Manual" (Denver, 1955).

2. R. L'HERMITE: "Idées actuelles sur la technologie du béton". Documentation Technique du Bâtiment et des Travaux Publics (París, 1955).

3. G. WERNER: "The effect of type of capping mate- rial on the compressive strength of concrete cylinders". Proc. A.S.T.M., 58, pp. 166-81 (1958).

4. A. M. NEVILLE: “Properties of Concrete” (1963).

5. VENUAT Y PAPADAKIS: "Control y ensayo de cementos, morteros y hormigones" (1966).

6. Normas UNE 7240 y 7242 : Fabricación, conservación y ensayo a compresión de probetas moldeadas de hormigón.

7. Normas A.S.T.M. C-31, C-39, C-192 y C-617.

8. A. DELIBES: "Análisis de la influencia de algunas variables en la extracción y ensayo a compresión de probetas-testigo de hormigón". Art. 462-3 de INFORMES DE LA CONSTRUCCION, 1974.

\section{résumé}

Etude de l'influence que les différents types de rectification exercent sur l'essai à la compression des éprouvettes en béton

A. Delibes Liniers,

ingénieur des Ponts et Chaussées

G. González Isabel, ingénieur technique

On expose les résultats de l'étude expérimentale des différentes techniques de rectification des éprouvettes en béton, qui sont toutes généralement admises comme correctes. On remarque des différences importantes de quelques systemes a d'autres. du est foulement recommandé d employer du soufre fondu pour des bétons jusqu'à lement quelques normes d'exécution qui ne ne figurent pas dans les différentes spécifications en vigueur.

\section{summary}

Study of the influence of various types of facing materials in concrete tests

A. Delibes Liniers, civil engineer

G. González Isabel, assistant civil engineer

The experimental results are given of tests on concrete specimens with various kinds of facings, all of which are generally considered valid. Considerable differences are observed between some systems and others and finally the use of cast sulphur is recommended for concretes with a strength of up to $500 \mathrm{~kg} / \mathrm{cm}^{2}$. Further, some workmanship regulations are establi. shed which do not figure in the various specifications in use at present.

\section{zusammenfassung}

Studium der Wirkung verschiedener Verkleidungsmaterialien bei Betondruckversuchen

A. Delibes Liniers,

ingenieur

G. González Isabel,

Es werden die Ergebnisse des experimentalen Studiums verschiedener Verkleidungsmethoden in Bezug auf Betonprobekörper präsentiert, wobei im allgemeinen alle Verfahren fur gültig gehalten werden. Es werden erhebliche Unterschiede zwischen
einigen Systemen und anderen beobachtet, und zuletzt wird der Gebrauch von gegos. senem Schwefel fur Betone mit eine
Festigkeit von bis auf $500 \mathrm{kp} / \mathrm{cm}^{2}$ empfoh len. Weiterhim werden Ausführungsnormen. die in den zur Zeit verwendeten Vorschriften nicht vorkommen, aufgestellt. 